Research Article

\title{
DFT Study on Adiabatic and Vertical Ionization Potentials of Graphene Sheets
}

\author{
Igor K. Petrushenko \\ Physical and Technical Institute, Irkutsk State Technical University, 83 Lermontov Street, Irkutsk 664074, Russia \\ Correspondence should be addressed to Igor K. Petrushenko; igor.petrushenko@istu.edu
}

Received 31 December 2014; Accepted 18 February 2015

Academic Editor: Richard Hennig

Copyright (c) 2015 Igor K. Petrushenko. This is an open access article distributed under the Creative Commons Attribution License, which permits unrestricted use, distribution, and reproduction in any medium, provided the original work is properly cited.

\begin{abstract}
Adiabatic and vertical ionization potentials (IPs) of finite-size graphene sheets as a function of size were determined by using density functional theory. In the case of graphene a very moderate gap between vertical and adiabatic IPs was observed, whereas for coronene molecule as a model compound these values differ considerably. The ionization process induces large changes in the structure of the studied sheets of graphene; "horizontal" and "vertical" bond lengths have different patterns of alternation. It was also established that the HOMO electron density distribution in the neutral graphene sheet affects its size upon ionization. The evolution of IPs of graphene sheets towards their work functions was discussed.
\end{abstract}

\section{Introduction}

The studies focusing on carbon allotropes, such as fullerenes [1], carbon nanotubes (CNTs) [2], and graphene [3], are a great challenge to scientists worldwide. Since 1986 extensive investigations on fullerenes and afterwards on CNTs have been conducted both theoretically and experimentally [46]. The last decade was the period of the rise of graphene. Its physical, chemical, structural, mechanical, and electronic properties were studied in detail [7-13].

Surprisingly, ionized graphene has received little attention. There are only a few papers, to the best of our knowledge, devoted to such investigations. Thus, Watanabe's group investigated Auger ionization in graphene nanoribbons (GNR) $[14,15]$. Ghadiry et al. studied the ionization mechanism theory in GNR [16]. Owens in his work studied electronic properties, band gap, and ionization potentials (IPs) of GNRs with various lengths [17].

Only two papers $[17,18]$ were devoted to the determination of IPs of graphene by the up-to-date density functional theory (DFT) technique; however, Owens studied only vertical ionization potential (IPV), whereas adiabatic ionization potential (IPA) was beyond the scope of his work. Baldoni's group used Koopmans' theorem to evaluate IPVs of GNRs. It is a matter of common knowledge that IPA and IPV of the same molecule are commonly close, but two different scenarios are in the basis of these two physical quantities and one must distinguish them. Generally, the first ionization potential approximates the work function (WF) providing useful information about the electron emitting behavior of the studied structures.

Also, we should emphasize that the transition of graphene from neutral to radical cation state is in close connection with the changes of its electron structure, which, in turn, improve the reactivity. Such activation can expand the limits of organic synthesis and allows one to obtain aim products selectively, more efficiently, and under nonrigid conditions.

The short list of aforementioned papers demonstrates that there is a demand to study both IPV and IPA of graphene. In addition, it is worth studying structural changes that arise in ionized graphene sheets, because the removal of an electron from a molecular orbital very probably causes large changes in the perfect framework of graphene.

Inspired by the above considerations as well as the recent success in precise cutting of graphene sheets [19], in this paper, we investigate electronic and structural properties of finite-sized graphene sheets by techniques based on the DFT, which has been successfully applied to predict many properties of graphene with high reliability and less computational efforts than the majority of the traditional ab initio methods. 
TABLE 1: Experimental and theoretical data on IPA and IPV of coronene.

\begin{tabular}{lcccccc}
\hline \multirow{2}{*}{ Compound } & \multicolumn{2}{c}{ B3LYP/SVP } & \multicolumn{2}{c}{ PBE0/SVP } & \multicolumn{3}{c}{ Experiment } \\
& IPA & IPV & IPA & IPV & IPA & IPV \\
\hline Coronene & 6.930 & 6.990 & \multirow{2}{*}{7.146} & 7.210 & $\begin{array}{c}7.26 \pm 0.05[27] ; 7.29 \\
{[28] ; 7.44[29] ; 7.64[30]}\end{array}$ & $7.29[31] ; 7.34[32]$ \\
\hline
\end{tabular}

\section{Computational Details}

The models $\mathrm{C}_{30} \mathrm{H}_{14}(3 \times 3,3$ aromatic rings in row and line, 9 rings in total), $\mathrm{C}_{48} \mathrm{H}_{18}(4 \times 4), \mathrm{C}_{70} \mathrm{H}_{22}(5 \times 5), \mathrm{C}_{96} \mathrm{H}_{26}$ $(6 \times 6)$, and $\mathrm{C}_{126} \mathrm{H}_{30}(7 \times 7)$ were used as neutral finitesize sheets of graphene. The ends of all studied models were passivated with hydrogen atoms to avoid the dangling. Orca 2.9.1 molecular modeling software [20] was used to perform DFT calculations on the investigated systems.

To validate our results we used a coronene molecule $\mathrm{C}_{24} \mathrm{H}_{12}$ as a well-studied model compound. Coronene was proved to be the smallest molecule that perfectly resembles graphene properties [21-23]. First, we calculated IPV and IPA of coronene using two hybrid functionals B3LYP and PBE0 that were employed in many research studies in which many properties of carbon nanotubes and graphene were studied, for example, [11, 24, 25]. The method used for obtaining IPA and IPV values is described below in this section. In all calculations we involved SVP (split-valence polarization) basis set, which is equivalent to the commonly used $6-31 \mathrm{G}^{*}$ basis set [26]. In SVP the inner shell atomic orbitals are described by a single basis function; two basis functions are provided for each valence shell atomic orbital, augmented by a set of polarization functions [26]. Second, we compared our theoretical predictions and available experimental evidence (Table 1).

Experimental results on IPV of Clar and Schmidt [31] as well as Boschi and Schmidt [32] are quite close: 7.29 and $7.34 \mathrm{eV}$, respectively. The great dispersion exists in the case of IPA: earlier works of Kuroda [30], Birks, and Slifkin [29] gave the following values: 7.64 and $7.44 \mathrm{eV}$, respectively. The subsequent investigators obtained smaller values: $7.26 \pm 0.05$ [27] and $7.29 \mathrm{eV}$ [28].

One can see that, by definition, calculated IPV of coronene is higher than its IPA (Table 1). B3LYP calculations give smaller IP values than does PBE0. Although it seems that both functionals slightly underestimate IP values, PBE0 produces more reliable results. Therefore, in the rest of the paper we involve only PBE0/SVP calculations.

The IPAs of the graphene sheets were determined by applying the following procedures. First, the total energies of a neutral sheet $E(G)$ and a +1 charge radical cation $E\left(G^{\bullet+}\right)$ of the same sheet were calculated. The ionization energy was then obtained by subtracting the total energy of the neutral sheet from the energy of the +1 charge radical cation:

$$
\mathrm{IPA}=E\left(G^{\bullet+}\right)-E(G)
$$

In order to obtain IPVs, we calculated the total energies of the ionic states of graphene sheets, using the optimized geometry of the neutral system; then we subtracted the total energy of the neutral from the energy of the radical cation of the graphene sheet obtained at the geometry of neutral:

$$
\mathrm{IPV}=E\left(G_{n}^{\bullet+}\right)-E(G)
$$

where $E\left(G^{\bullet+}{ }_{n}\right)$ is the total energy calculated at the same equilibrium geometry as the neutral graphene sheet.

The work function of the systems considered here is obtained from the Fermi energy:

$$
E_{F}=E_{\mathrm{HOMO}}+\frac{E_{g}}{2},
$$

where $E_{\mathrm{HOMO}}$ is the energy of the highest occupied molecular orbital and $E_{g}$ is the band gap of the system.

\section{Results and Discussion}

3.1. Ionization Potentials of Finite-Size Graphene Sheets. To study the influence of graphene sheets size on their electronic structures we have chosen the following models: $\mathrm{C}_{30} \mathrm{H}_{14}(3 \times$ 3), $\mathrm{C}_{48} \mathrm{H}_{18}(4 \times 4), \mathrm{C}_{70} \mathrm{H}_{22}(5 \times 5), \mathrm{C}_{96} \mathrm{H}_{26}(6 \times 6)$, and $\mathrm{C}_{126} \mathrm{H}_{30}(7 \times 7)$. Such a moderate size of sheets was chosen to expressly investigate quantum size effects that become especially explicit in such systems.

Figure 1 presents the studied graphene sheets as well as a coronene molecule which is used for comparison purposes.

With obtained information concerning IPA and IPV, we compared two series of values from Table 2 .

Since the relaxed geometry of the radical cation of graphene is sure of lower energy than its unrelaxed geometry corresponding to neutral graphene, IPV > IPA for all studied models. Although the difference between IPV and IPA seems rather small, we should separate these two definitions of IPs because two different scenarios underlie the basis of these values. In the examination of infinite graphene, we can very likely use any IP definition. Also, we took into consideration available data on IP calculations of singlewalled CNTs (SWCNTs) which may be regarded as "rolled" graphene sheets. Unfortunately, many authors did not state clearly whether their values are adiabatic or vertical [33-35]. The remarkable exceptions are the works $[24,36]$. There are two papers devoted to the IPs determination of graphene $[17,18]$ and they contain data on IPVs only. Moreover, Baldoni's work [18] employs Koopmans' theorem to obtain IP values which, though very convenient, gives only rough approximations. Additionally, in this work, we use different size of the graphene sheets in comparison with the latter papers. Therefore, it is unreasonable to directly match our IPs predictions and literature evidence on both SWCNTs and graphene. On the other hand, we can compare general trends 


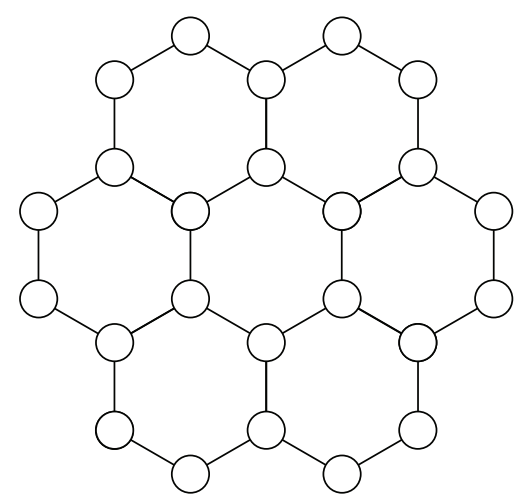

Coronene

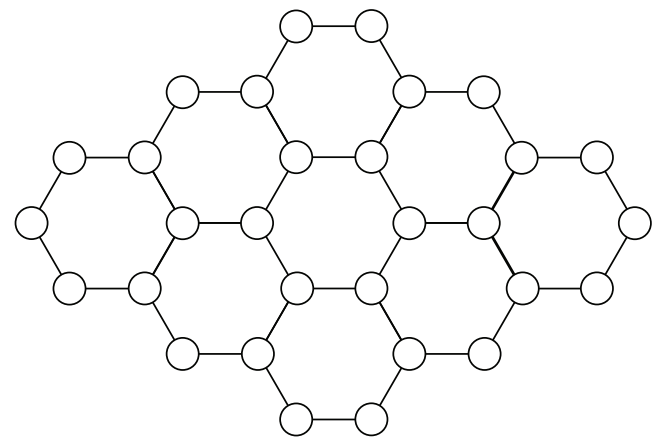

$3 \times 3$

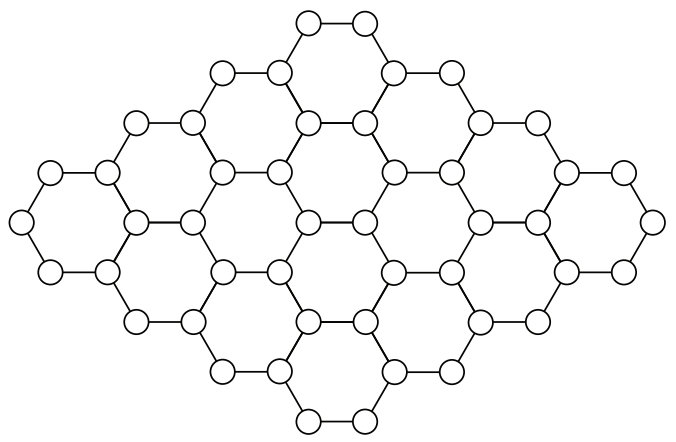

$4 \times 4$

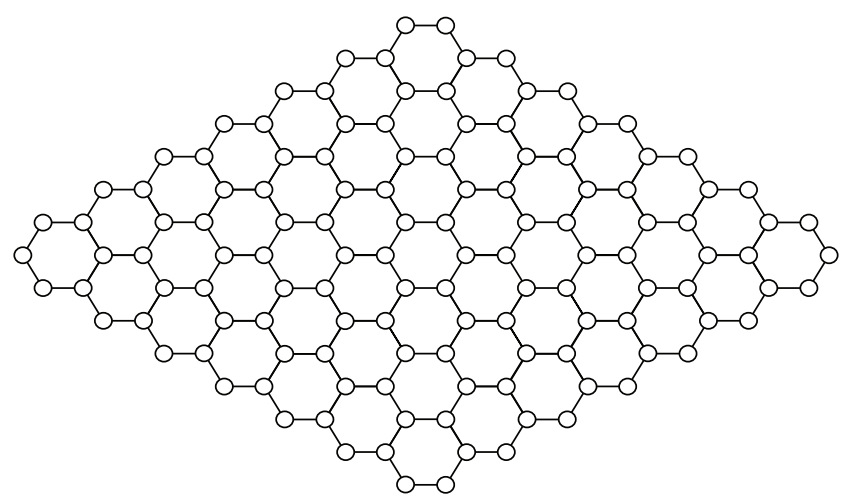

$7 \times 7$

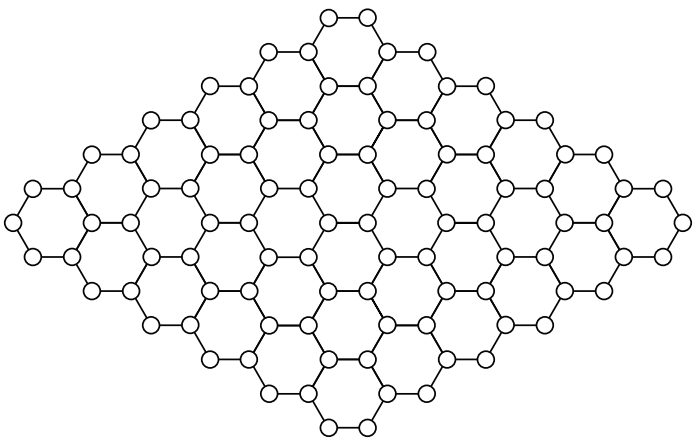

$6 \times 6$

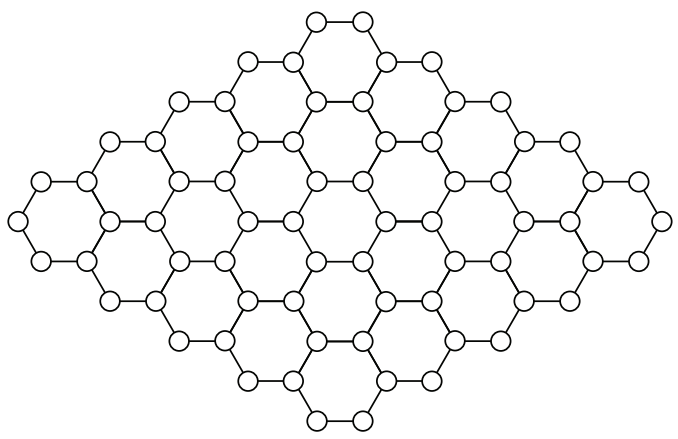

$5 \times 5$

FIGURE 1: Six optimized structures studied in this work: coronene as the simplest model of graphene and five finite-size graphene sheets from $3 \times 3$ to $7 \times 7$. Hydrogen atoms omitted for clarity.

in changing of IPs with the outcomes of the aforementioned papers.

Thus, $\mathrm{C}_{30} \mathrm{H}_{16}, \mathrm{C}_{46} \mathrm{H}_{18}$, and $\mathrm{C}_{62} \mathrm{H}_{22}$ models from Owens' work have the following IPV values: 5.8, 5.2, and $4.75 \mathrm{eV}$, respectively. It is in a good accordance with our results (Table 2). One can observe the similar trend: monotonic decrease in the IP value with the increase in nanocluster size. When the number of carbons in the structure is even, the sheets demonstrate similar IPs behavior, whereas when it is odd the IPs versus length curve show a clear peak and then a gradual decrease in IP values. Baldoni's paper demonstrates the following value for the model consisting of 30 carbon atoms: $7.3 \mathrm{eV}$ [18]. It is the only "armchair" model which has an approximately equal number of carbon atoms as compared with the present work models. This overstated value of IPV was ascribed to the usage of Koopmans' theorem. In spite of a very limited range of IP values for armchair models, we can, however, compare our data with the trends in the changing of IPVs of "chiral" and "zigzag" models. It turned out that Baldoni's results have the same trend: monotonic decrease in IPVs. The plots do not allow the peculiar peak observed by Owens to be recognized.

SWCNTs, in turn, demonstrate the opposite behavior of their IPs. One can see pronounced oscillations of both IPAs and IPVs with the period of $3[24,36]$. The origin of these oscillations was suggested elsewhere [24,37]. It is clear that to follow this pattern the studied graphene sheets should form entirely identical structures. However, the general decrease in 
TABLe 2: Properties of optimized graphene sheets as described in the paper.

\begin{tabular}{|c|c|c|c|c|c|}
\hline Graphene sheet & Length $(G)^{\mathrm{a}}, \AA$ & Length $\left(G^{\cdot+}\right), \AA$ & $\Delta L^{\mathrm{b}}, \AA$ & IPA, eV & IPV, eV \\
\hline $3 \times 3$ & 3.656 & 3.683 & 0.027 & 6.10 & 6.15 \\
\hline $4 \times 4$ & 6.100 & 6.117 & 0.017 & 5.46 & 5.49 \\
\hline $5 \times 5$ & 8.607 & 8.593 & 0.014 & 5.08 & 5.09 \\
\hline $6 \times 6$ & 11.056 & 11.073 & 0.017 & 4.85 & 4.86 \\
\hline $7 \times 7$ & 13.507 & 13.518 & 0.011 & 3.76 & 3.85 \\
\hline
\end{tabular}

${ }^{\mathrm{a}}$ Length $(G)$ is the length of the graphene sheet as described in Figure $3 ;{ }^{\mathrm{b}} \Delta L=$ Length $\left(G^{\circ+}\right)-$ Length $(G)$.

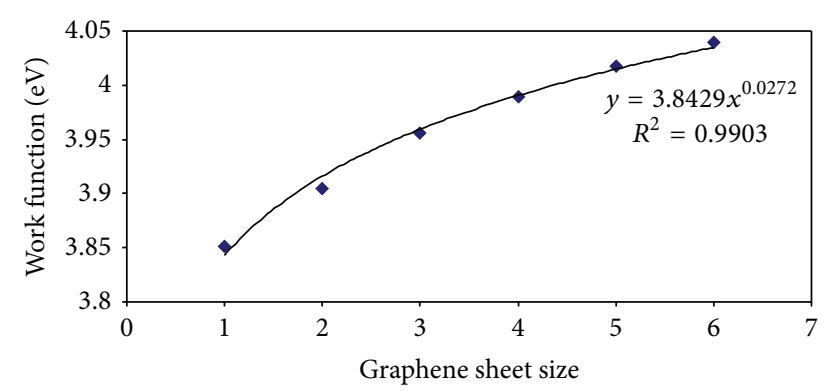

FIGURE 2: The work function versus graphene sheet size plot $(1-3 \times$ $3 ; 2-4 \times 4 ; 3-5 \times 5 ; 4-6 \times 6 ; 5-7 \times 7 ; 6-8 \times 8)$.

IPs with increasing size of SWCNTs is sure to confirm our IP calculations as well as conclusions about infinite graphene behavior.

3.2. Work Function of Graphene. Our next aim is to study the evolution of the IPs of finite-size graphene sheets to the WF of "infinite" graphene. We first address the work functions of small graphene clusters with sizes ranging from $c a .7 \times 7 \AA$ to $18 \times 18 \AA$ (from $3 \times 3$ to $8 \times 8$ models). The calculated WFs are presented in Figure 2.

The data analysis shows that the WF increases with the increasing graphene sheet size (Figure 2). On going from "small" to "large" models the relative changes of the WF diminish. As far as we know, there are no direct experimental or theoretical measurements on the WFs of individual finitesize graphene sheets. Therefore, it is worth studying qualitatively the critical size of a polyaromatic hydrocarbon that gradually becomes real graphene. To assess that, we extrapolate our data with a power function (Figure 2); excellent correlation coefficient $\left(R^{2}=0.99\right)$ is observed.

Extrapolation towards a larger size $(c a .790 \times 790 \AA$ or $330 \times 330$ sheet) gives the WF of $4.50 \mathrm{eV}$, which is close to the WFs of graphene (4.5 [38], 4.55 [39] eV). Thus, in terms of electronic properties, we can conclude that the 330 $\times 330$ sheet is large enough to describe well the properties of "infinite" graphene. It practically corresponds with "the upper limit of nanotechnologies." Further extrapolation gives us the following critical point of transition from graphene to bulky graphite: $c a .8500 \times 8500 \AA(3550 \times 3550)$ sheet demonstrates a WF of $4.80 \mathrm{eV}$, which is very close to the WFs for the graphite $(4.8 \mathrm{eV}$ [40], $4.91 \mathrm{eV}$ [41]).

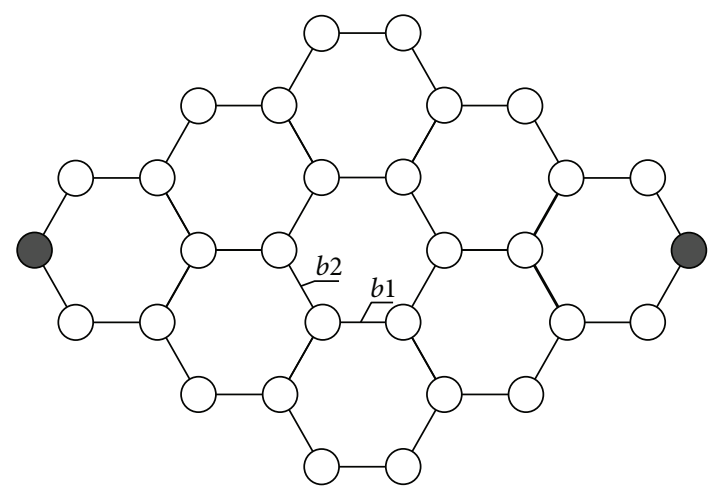

FIgURE 3: The model used herein to define structural parameters of graphene sheets: "horizontal" (b1) and "vertical" (b2) bond lengths. Terminate carbon atoms, between which the length of the sheet is measured, are colored in black. Hydrogen atoms omitted for clarity.

3.3. Structures of Graphene Sheets. To study the influence of graphene sheets ionization on their structures we, firstly, should define some useful parameters. There are two types of bonds in graphene sheets, one "horizontal" (b1) and one "vertical" (b2) (Figure 3).

It should be noted that we use such notations to emphasize the basic difference of these bonds. Actually, the angle between $b 2$ and the ordinate axis is $c a .30^{\circ}$. To define the influence of ionization on geometrical parameters of the studied graphene sheets, we measure the lengths of these clusters between the terminate carbon atoms (Figure 3 ). Further, we will review $7 \times 7$ graphene sheet as the most interesting one among those studied.

First, we need to say that ionization leaves $\mathrm{C}-\mathrm{H}$ bond lengths intact. We do not include these bonds into further consideration. A large bond alternation is observed for "horizontal" $b 1$ (Figure 4). They were established to be symmetric relative to the graphene sheet centre. Similar observations were made in the previous studies on CNTs [24, 35]. The centre bond lengths are much closer than peripheral bonds to the value of $1.42 \AA$ ( $\mathrm{C}-\mathrm{C}$ bond length of pristine infinite graphene). The terminate $\mathrm{C}$-C bonds undergo the influence of hydrogen atoms as well as the edge effects. Ionization induces large changes in the framework of the sheets. Almost every $\mathrm{C}-\mathrm{C}$ bond becomes longer as an electron is being removed which makes the bonds weaker. Alternation pattern is also changed; we cannot observe any symmetry in bond lengths. 

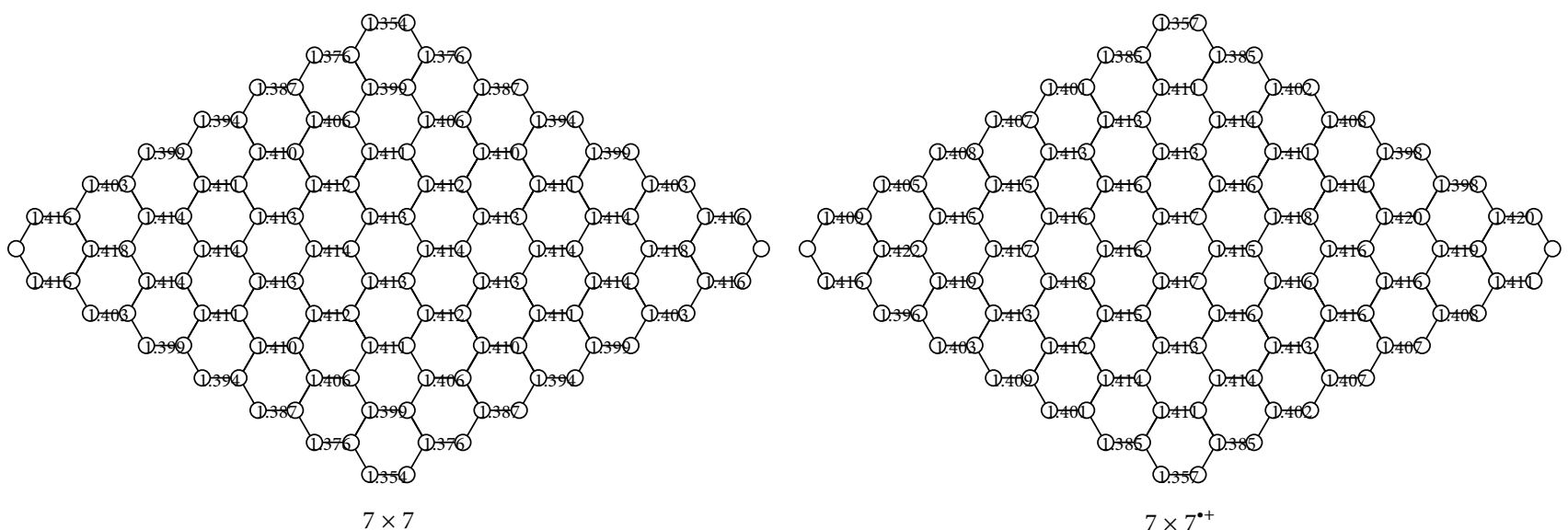

FIGURE 4: Optimized $b 1$ bond lengths (in angstroms) of neutral $7 \times 7$ and its radical cation $7 \times 7^{\bullet+}$. Hydrogen atoms omitted for clarity.
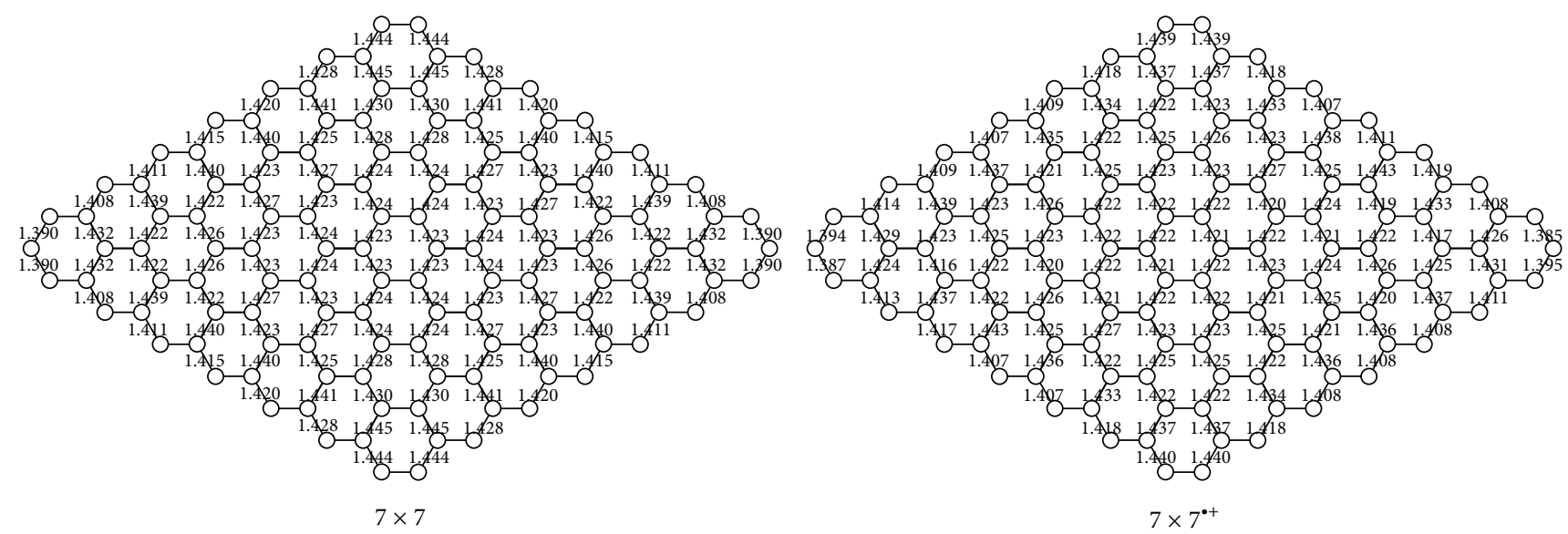

FIGURE 5: Optimized $b 2$ bond lengths (in angstroms) for both neutral $7 \times 7$ and its radical cation $7 \times 7^{\circ+}$. Hydrogen atoms omitted for clarity.

The "vertical" $b 2$ bond lengths show the same behavior: neutral graphene sheet exhibits clear symmetry of $b 2$, whereas its radical cation has a lack of symmetry (Figure 5). The majority of $b 1$ bonds become longer on ionization. The behavior of $b 2$ is opposite as we can see both the elongation and the abridgement equally. To shed the light on this problem, we take into consideration the highest occupied molecular orbital (HOMO) of the graphene sheet (Figure 6). The relative elongation or the abridgement of $b 1$ or $b 2$ is probably caused by the shape of the HOMO from which the electron is being removed. As we can see, the distribution of the electron cloud is not uniform; there are distinct minima and maxima on the graphene surface (Figure 6). It is displaced from the top and the bottom of the sheet, which leads to the nonuniform structural changes of ionized graphene.

As a whole, the process of ionization increases the length of the graphene sheet as a cluster; besides, this effect is strongly pronounced in the case of "small" sheets. It is natural as the whole number of electrons increases on going from $3 \times 3$ to $7 \times 7$ models and the impact of a single electron to the cluster's electron shape becomes smaller. In the case of infinite sheets of graphene we expect zero contribution from one electron. However, the recent experiments have demonstrated that the "atomically precise" graphene sheets became a reality $[19,42]$. Such sheets were employed in modern graphene nanoelectromechanical systems (GNEMS) to enhance their properties [43]; therefore we should keep in mind that a finite-size graphene sheet may change its size upon ionization.

\section{Conclusion}

We investigated IPA and IPV for a series of graphene sheets as a function of their size by means of the PBE0/SVP approach. Our calculations reveal that the size of the graphene sheet affects its IPs values. In contrast to coronene, graphene shows only marginal difference between IPA and IPV that approves its greater structural stiffness. It looks very possible that in the case of real graphene both types of IPs become equal. It was established that the size of studied sheets undergoes changes upon ionization and in the case of "small" sheets these relative changes are significant. This interesting effect of graphene elongation, most probably, depends on the HOMO electron density distribution in graphene. Finally, in terms 


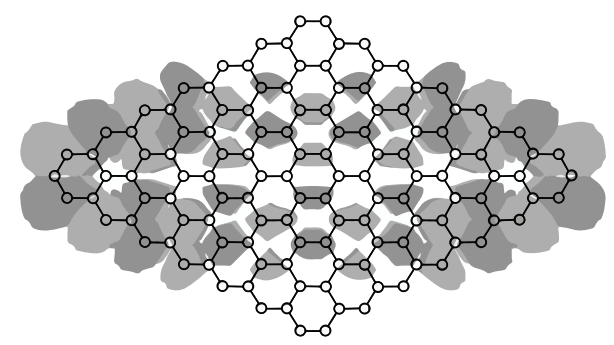

FIgure 6: The highest occupied molecular orbital (HOMO) of neutral $7 \times 7$. Hydrogen atoms omitted for clarity.

of electronic properties, we have found that graphene, when reaching the critical size of approximately $790 \times 790 \AA$, can be considered as real graphene.

\section{Conflict of Interests}

The author declares that there is no conflict of interests regarding the publication of this paper.

\section{Acknowledgments}

The reported study was supported by RFBR, research project no. 14-03-31231 мол_а. Generous allotment of computational time from Supercomputing Center of Novosibirsk State University (http://www.nsu.ru/, http://www.nusc.ru/) is gratefully acknowledged. The author thanks the SAIA (http://www.saia.sk/en) for support.

\section{References}

[1] H. W. Kroto, J. R. Heath, S. C. O’Brien, R. F. Curl, and R. E. Smalley, “C60: buckminsterfullerene," Nature, vol. 318, no. 6042, pp. 162-163, 1985.

[2] S. Iijima, "Helical microtubules of graphitic carbon," Nature, vol. 354, no. 6348, pp. 56-58, 1991.

[3] K. S. Novoselov, A. K. Geim, S. V. Morozov et al., "Electric field effect in atomically thin carbon films," Science, vol. 306, no. 5696, pp. 666-669, 2004.

[4] I. K. Petrushenko and N. A. Ivanov, "DFT study of the structural and mechanical properties of oxydated single-walled carbon nanotubes," Fullerenes Nanotubes and Carbon Nanostructures, vol. 22, no. 5, pp. 405-412, 2014.

[5] I. K. Petrushenko and N. A. Ivanov, "DFT study of the elastic properties of pristine and moderately fluorinated singlewalled carbon nanotubes," Fullerenes Nanotubes and Carbon Nanostructures, vol. 22, no. 9, pp. 781-788, 2014.

[6] M. Yamada, T. Akasaka, and S. Nagase, "Carbene additions to fullerenes," Chemical Reviews, vol. 113, no. 9, pp. 7209-7264, 2013.

[7] J. O. Sofo, A. S. Chaudhari, and G. D. Barber, "Graphane: a two-dimensional hydrocarbon," Physical Review B-Condensed Matter and Materials Physics, vol. 75, no. 15, Article ID 153401, 2007.

[8] D. W. Boukhvalov and M. I. Katsnelson, "Chemical functionalization of graphene," Journal of Physics Condensed Matter, vol. 21, no. 34, Article ID 344205, 2009.
[9] C. N. R. Rao, A. K. Sood, K. S. Subrahmanyam, and A. Govindaraj, "Graphene: the new two-dimensional nanomaterial," Angewandte Chemie, vol. 48, no. 42, pp. 7752-7777, 2009.

[10] P. A. Denis and F. Iribarne, "The 1,3 dipolar cycloaddition of azomethine ylides to graphene, single wall carbon nanotubes, and C60," International Journal of Quantum Chemistry, vol. 110, pp. 1764-1771, 2010.

[11] I. K. Petrushenko, "[2+1] Cycloaddition of dichlorocarbene to finite-size graphene sheets: DFT study," Monatshefte fur Chemie, vol. 145, no. 6, pp. 891-896, 2014.

[12] Y. Shao, J. Wang, H. Wu, J. Liu, I. A. Aksay, and Y. Lin, "Graphene based electrochemical sensors and biosensors: a review," Electroanalysis, vol. 22, no. 10, pp. 1027-1036, 2010.

[13] C. Xu, B. Xu, Y. Gu, Z. Xiong, J. Sun, and X. S. Zhao, "Graphenebased electrodes for electrochemical energy storage," Energy and Environmental Science, vol. 6, no. 6, pp. 1388-1414, 2013.

[14] S. Konabe, N. Onoda, and K. Watanabe, "Auger ionization in armchair-edge graphene nanoribbons," Physical Review BCondensed Matter and Materials Physics, vol. 82, no. 7, Article ID 073402, 2010.

[15] N. Onoda, S. Konabe, T. Yamamoto, and K. Watanabe, "Auger ionization in carbon nanotubes and graphene nanoribbons under laser irradiation," Physica Status Solidi (C), vol. 8, no. 2, pp. 570-572, 2011.

[16] M. Ghadiry, A. B. A. Manaf, M. Nadi, M. Rahmani, and M. T. Ahmadi, "Theory of ionization mechanism in graphene nanoribbons," Journal of Computational and Theoretical Nanoscience, vol. 9, no. 12, pp. 2190-2192, 2012.

[17] F. J. Owens, "Electronic and magnetic properties of armchair and zigzag graphene nanoribbons," Journal of Chemical Physics, vol. 128, no. 19, Article ID 194701, 2008.

[18] M. Baldoni, A. Sgamellotti, and F. Mercuri, "Electronic properties and stability of graphene nanoribbons: an interpretation based on Clar sextet theory," Chemical Physics Letters, vol. 464, no. 4-6, pp. 202-207, 2008.

[19] Y.-C. Chen, D. G. de Oteyza, Z. Pedramrazi, C. Chen, F. R. Fischer, and M. F. Crommie, "Tuning the band gap of graphene nanoribbons synthesized from molecular precursors," ACS Nano, vol. 7, no. 7, pp. 6123-6128, 2013.

[20] F. Neese, “The ORCA program system," Wiley Interdisciplinary Reviews: Computational Molecular Science, vol. 2, no. 1, pp. 7378, 2012.

[21] J. Kysilka, M. Rubeš, L. Grajciar, P. Nachtigall, and O. Bludský, "Accurate description of argon and water adsorption on surfaces of graphene-based carbon allotropes," Journal of Physical Chemistry A, vol. 115, no. 41, pp. 11387-11393, 2011.

[22] P. Lazar, F. Karlický, P. Jurecka et al., "Adsorption of small organic molecules on graphene," Journal of the American Chemical Society, vol. 135, no. 16, pp. 6372-6377, 2013.

[23] E. Voloshina, D. Usvyat, M. Schütz, Y. Dedkov, and B. Paulus, "On the physisorption of water on graphene: a CCSD(T) Study," Physical Chemistry Chemical Physics, vol. 13, no. 25, pp. 1204112047, 2011.

[24] I. K. Petrushenko and N. A. Ivanov, "Ionization potentials and structural properties of finite-length single-walled carbon nanotubes: DFT study," Physica E: Low-Dimensional Systems and Nanostructures, vol. 54, pp. 262-266, 2013.

[25] I. K. Petrushenko and N. A. Ivanov, "Structural and electronic properties of finite-length single-walled carbon and silicon carbide nanotubes: DFT study," Modern Physics Letters B, vol. 27, no. 29, Article ID 1350210, 2013. 
[26] A. Schäfer, C. Huber, and R. Ahlrichs, "Fully optimized contracted Gaussian basis sets of triple zeta valence quality for atoms Li to Kr," The Journal of Chemical Physics, vol. 100, no. 8, pp. 5829-5835, 1994.

[27] M. Meot-Ner, "Ion thermochemistry of low-volatility compounds in the gas phase. 3. Polycyclic aromatics: ionization energies, proton and hydrogen affinities. Extrapolations to graphite," Journal of Physical Chemistry, vol. 84, no. 21, pp. 27162723, 1980.

[28] E. Clar, J. M. Robertson, R. Schlögl, and W. Schmidt, "Photoelectron spectra of polynuclear aromatics. 6. Applications to structural elucidation: 'Circumanthracene', Journal of the American Chemical Society, vol. 103, no. 6, pp. 1320-1328, 1981.

[29] J. B. Birks and M. A. Slifkin, " $\pi$-electronic excitation and ionization energies of condensed ring aromatic hydrocarbons," Nature, vol. 191, no. 4790, pp. 761-764, 1961.

[30] H. Kuroda, "Ionization potentials of polycyclic aromatic hydrocarbons," Nature, vol. 201, no. 4925, pp. 1214-1215, 1964.

[31] E. Clar and W. Schmidt, "Correlations between photoelectron and phosphorescence spectra of polycyclic hydrocarbons," Tetrahedron, vol. 32, no. 21, pp. 2563-2566, 1976.

[32] R. Boschi and W. Schmidt, "Photoelectron spectra of polycyclic aromatic hydrocarbons. Pyrene and coronene," Tetrahedron Letters, vol. 13, no. 25, pp. 2577-2580, 1972.

[33] F. Buonocore, F. Trani, D. Ninno, A. Di Matteo, G. Cantele, and G. Iadonisi, "Ab initio calculations of electron affinity and ionization potential of carbon nanotubes," Nanotechnology, vol. 19, no. 2, Article ID 025711, 2008.

[34] D. A. Buzatu, A. S. Biris, A. R. Biris, D. M. Lupu, J. A. Darsey, and M. K. Mazumder, "Electronic properties of single-wall carbon nanotubes and their dependence on synthetic methods," IEEE Transactions on Industry Applications, vol. 40, no. 5, pp. 12151219, 2004.

[35] A. Galano, "On the influence of diameter and length on the properties of armchair single-walled carbon nanotubes: a theoretical chemistry approach," Chemical Physics, vol. 327, no. 1, pp. 159-170, 2006.

[36] Z. Zhou, M. Steigerwald, M. Hybertsen, L. Brus, and R. A. Friesner, "Electronic structure of tubular aromatic molecules derived from the metallic $(5,5)$ armchair single wall carbon nanotube," Journal of the American Chemical Society, vol. 126, no. 11, pp. 3597-3607, 2004.

[37] Y. Matsuo, K. Tahara, and E. Nakamura, “Theoretical studies on structures and aromaticity of finite-length armchair carbon nanotubes," Organic Letters, vol. 5, no. 18, pp. 3181-3184, 2003.

[38] C. Christodoulou, A. Giannakopoulos, M. V. Nardi et al., "Tuning the work function of graphene-on-quartz with a high weight molecular acceptor," Journal of Physical Chemistry C, vol. 118, no. 9, pp. 4784-4790, 2014.

[39] V. Barone, J. E. Peralta, J. Uddin, and G. E. Scuseria, "Screened exchange hybrid density-functional study of the work function of pristine and doped single-walled carbon nanotubes," The Journal of Chemical Physics, vol. 124, no. 2, Article ID 024709, 2006.

[40] M. Shiraishi and M. Ata, "Work function of carbon nanotubes," Carbon, vol. 39, no. 12, pp. 1913-1917, 2001.

[41] J. Zhao, J. Han, and J. Lu, "Work functions of pristine and alkalimetal intercalated carbon nanotubes and bundles," Physical Review B, vol. 65, no. 19, Article ID 193401, 4 pages, 2002.

[42] L. Ma, J. Wang, and F. Ding, "Recent progress and challenges in graphene nanoribbon synthesis," ChemPhysChem, vol. 14, no. 1, pp. 47-54, 2013.
[43] C. Chen and J. Hone, "Graphene nanoelectromechanical systems," Proceedings of the IEEE, vol. 101, no. 7, pp. 1766-1779, 2013. 

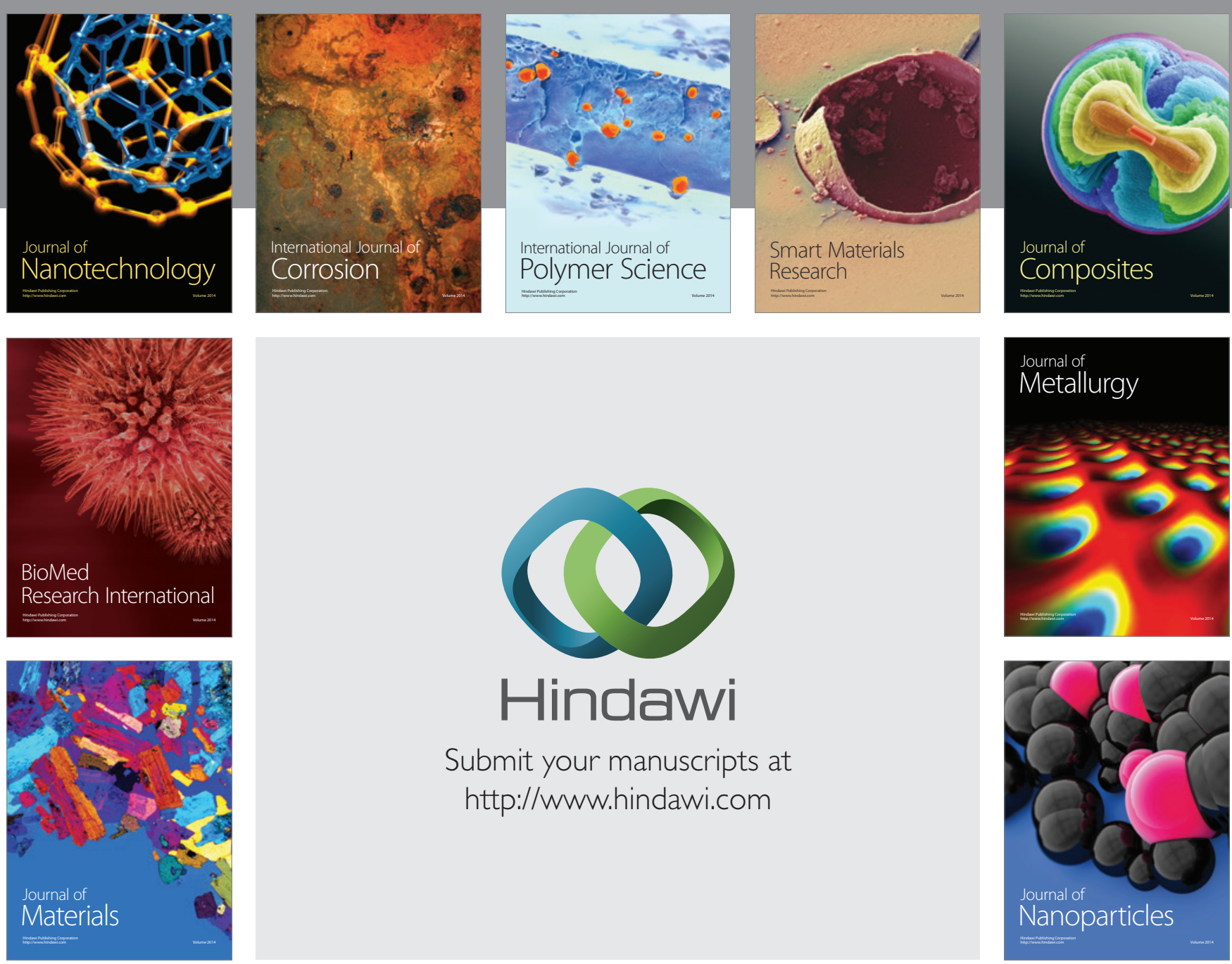

Submit your manuscripts at http://www.hindawi.com
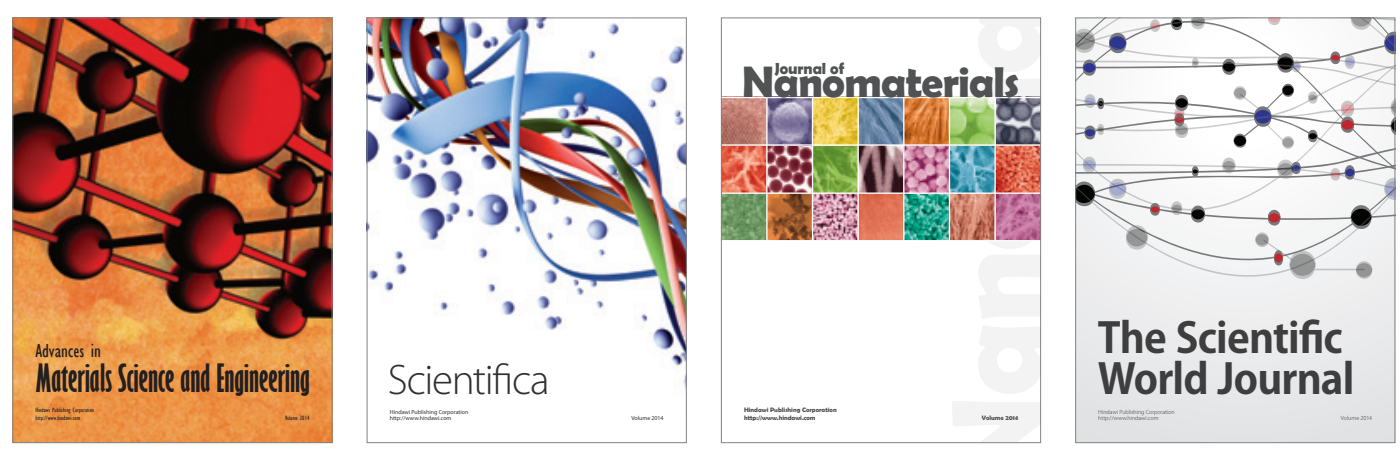

\section{The Scientific World Journal}
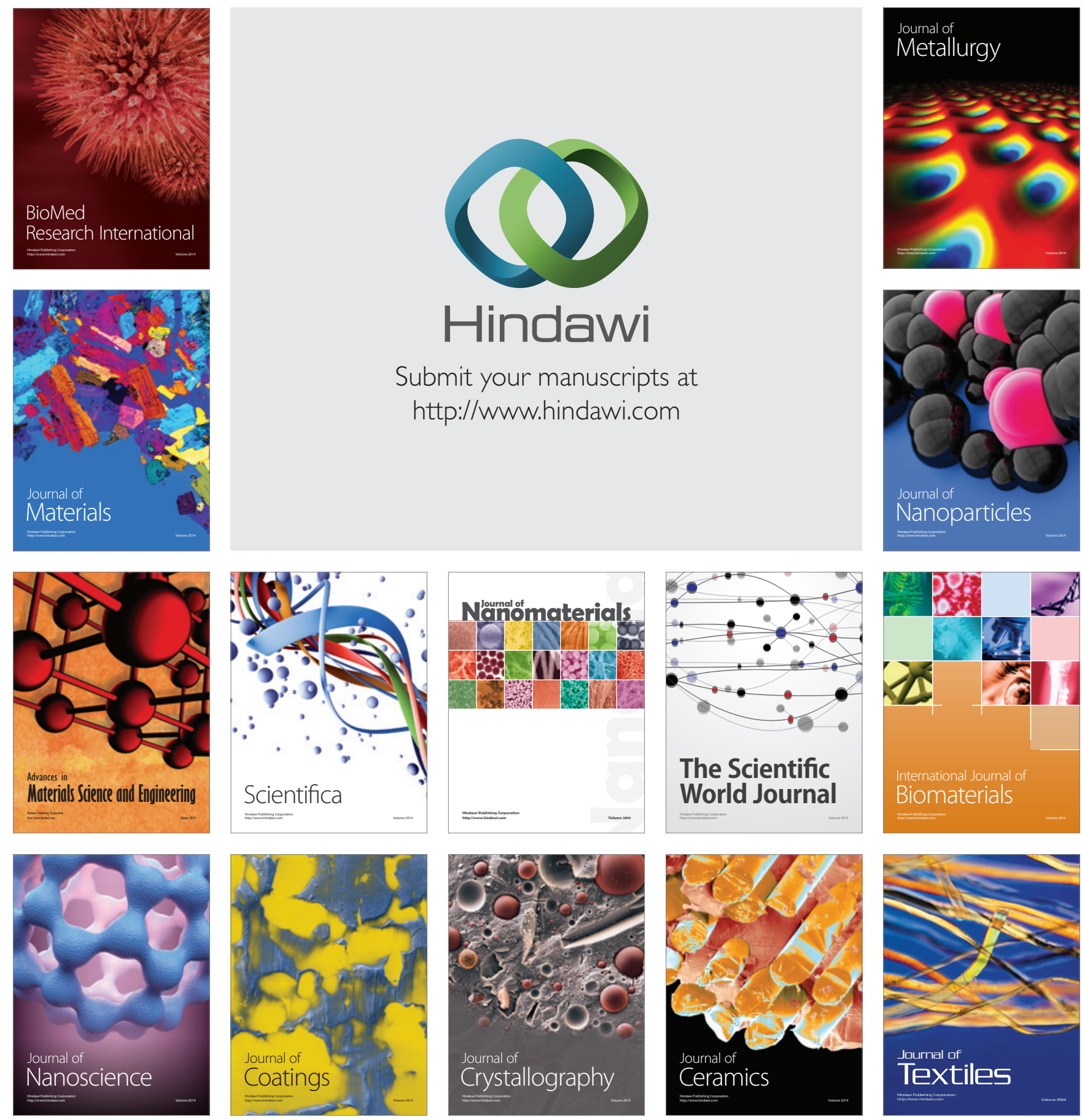